\title{
Spontaneous scalarization of self-gravitating magnetic fields
}

\author{
Yves Brihaye, ${ }^{1}$ Rogério Capobianco $\odot,{ }^{2}$ and Betti Hartmann ${ }^{2,3,4,5}$ \\ ${ }^{1}$ Service de Physique de l'Univers, Champs et Gravitation, Université de Mons, 7000 Mons, Belgium \\ ${ }^{2}$ Instituto de Física de São Carlos, Universidade de São Paulo, São Carlos, São Paulo 13560-970, Brazil \\ ${ }^{3}$ Institut für Physik, Carl-von-Ossietzky Universität Oldenburg, 26111 Oldenburg, Germany \\ ${ }^{4}$ Department of Physics and Earth Sciences, Jacobs University Bremen, 28759 Bremen, Germany \\ ${ }^{5}$ Department of Mathematics, University College London, \\ Gower Street, London WC1E 6BT, United Kingdom
}

(Received 2 April 2021; accepted 14 May 2021; published 9 June 2021)

\begin{abstract}
In this paper, we study the spontaneous scalarization of an extended, self-gravitating system which is static, cylindrically symmetric and possesses electromagnetic fields. We demonstrate that a real massive scalar field condenses on this Melvin magnetic universe solution when introducing a nonminimal coupling between the scalar field and (a) the magnetic field and (b) the curvature of the space-time, respectively. We find that in both cases, the solutions exist on a finite interval of the coupling constant and that solutions with a number of nodes $k$ in the scalar field are present. For case (a) we observe that the intervals of existence are mutually exclusive for different $k$.
\end{abstract}

DOI: 10.1103/PhysRevD.103.124020

\section{INTRODUCTION}

Multimessenger observations of compact objects allow one to test general relativity (GR) and its extensions to high precision now and in the future. As such, renewed interest in testing no-hair and/or uniqueness theorems for black holes has appeared. While work in the 1990s was mainly devoted to the construction of "hairy" black holes in the context of general relativity supplemented with nonlinear matter fields appearing in particle physics models, recent activity has focused on the extension of the gravity part of the model, e.g., by adding a nonminimal interaction between higher order curvature terms and extra gravitational fields; see e.g., [1,2] for reviews.

In a number of these extended gravity models new black hole solutions with nontrivial fields on the horizon that vanish asymptotically have been shown to exist. In fact, these new black hole solutions appear for specific intervals of the nonminimal coupling. Outside of this interval, the black hole solutions are equivalent to the standard black hole solutions that fulfill the no-hair theorems, i.e., are equivalent to either the Schwarschild, Reissner-Nordström or Kerr(-Newman) solution. In these extended models black holes are hence said to "scalarize spontaneously" [3-5] in the case of nonminimal coupling to a scalar field or "vectorize spontaneously" [6-9] in the case of nonminimal couplings to Abelian gauge fields.

However, the idea of spontaneous scalarization is not specific to black holes, but has been shown to appear also for other compact objects such as boson stars [10-12] and neutron stars [13]. It can also appear in extended systems, such as low density stars [14] and even the whole universe itself [15].

In this paper, we want to show that spontaneous scalarization exists also for the Melvin solution, an extended self-gravitating system that describes an electromagnetic field kept together by its own gravity [16] and that was shown to be stable in the context of general relativity [17]. Charged black holes embedded in such a magnetic universe have been studied recently [18] and it has been shown that they can carry minimally coupled, complex and ungauged scalar hair.

The electromagnetic field of the "pure" Melvin solution points in the direction of the symmetry axis and the solution is essentially characterized by the absolute value of this field on the symmetry axis. As such it is a cylindrically symmetric gravitating system (for a review see e.g., [19]). The most studied cylindrically symmetric extended selfgravitating system is surely the cosmic string, a topological defect that might have formed in the primordial universe [20]. However, magnetic fields in elongated regions were observed astrophysically in so-called radio relics. Radio relics are diffuse radio sources in galaxy clusters. These sources are not associated to any cluster galaxy [21] and have been categorized into three groups: radio gischt, radio phoenix and active galactic nucleus relics [22]. The radio gischt are mostly found in the outskirts of galaxies and are elongated arclike radio sources with sizes of up to $2 \mathrm{Mpc}$. Observations give support to the hypothesis that they trace shock fronts in which particles are accelerated via the diffuse shock acceleration mechanism and possess ordered magnetic fields with a few micro-Gauss strength 
(see e.g., [23]). As such they present probably the largest magnetic structures in the universe. One interesting case of gischtlike sources are so-called "double-relics." In this case two relics are diametrically located on both sides of the cluster center, see e.g., [24] and references therein. Due to the improvement in instrument sensitivity the number of detections of radio relics has grown dramatically in the last decade. Large cosmological simulations that include radio emissions from shocks suggest that these structures should form frequently, see e.g., [24,25] and references therein. Certainly, the Melvin magnetic universe can only be an idealized model for extended but finite magnetic field structures, but we believe that it can give qualitative ideas about the phenomenon which is easily trackable because of the fact that both the space-time as well as the magnetic field are given analytically. A full simulation of radio relics (including the gravitational field) is certainly a formidable task and beyond the aim of this paper.

Motivated by the existence of elongated and ordered magnetic fields in the universe, we study the EinsteinMaxwell model and add a massive, real scalar that is nonminimally coupled to the system. In order to understand the effects of the nonminimal coupling we study two different scenarios separately: (a) the nonminimal coupling to the electromagnetic field and (b) the nonminimal coupling to the Gauss-Bonnet curvature term. These two coupling options have been used extensively in the recent construction of black holes with scalar hair.

Our paper is organized as follows: In Sec. II, we give the model and Ansatz and also discuss the small scalar field limit. In Sec. III we present our results for the case of scalarmagnetic field coupling, while Sec. IV is concerned with the scalar-gravity case. We conclude in Sec. V.

\section{THE MODEL AND ANSATZ}

In this paper, we study a scalar-tensor gravity model with the following action [we use metric signature $(+---)$ ]:

$$
\begin{aligned}
S= & \int \mathrm{d}^{4} x \sqrt{-g}\left[\frac{\mathcal{R}}{16 \pi G}+\phi^{2}\left(\alpha F_{\mu \nu} F^{\mu \nu}+\gamma \mathcal{G}\right)\right. \\
& \left.+\frac{1}{2} \partial_{\mu} \phi \partial^{\mu} \phi-\frac{m^{2}}{2} \phi^{2}-\frac{1}{4} F_{\mu \nu} F^{\mu \nu}\right],
\end{aligned}
$$

where $\mathcal{R}$ is the Ricci scalar, $\mathcal{G}$ the Gauss-Bonnet term, $F_{\mu \nu}=\partial_{\mu} A_{\nu}-\partial_{\nu} A_{\mu}$ the field strength tensor of a $\mathrm{U}(1)$ gauge field $A_{\mu}$ and $\phi$ a real-valued scalar field with mass $m$ that is coupled to the Maxwell invariant $F_{\mu \nu} F^{\mu \nu}$ as well as the Gauss-Bonnet term $\mathcal{G}$ given by

$$
\mathcal{G}=\left(\mathcal{R}^{\mu \nu \rho \sigma} \mathcal{R}_{\mu \nu \rho \sigma}-4 \mathcal{R}^{\mu \nu} \mathcal{R}_{\mu \nu}+\mathcal{R}^{2}\right)
$$

via the couplings $\alpha$ and $\gamma$, respectively. The equations of motion then read

$$
\square \phi+\left(2 \alpha F_{\mu \nu} F^{\mu \nu}+2 \gamma \mathcal{G}-m^{2}\right) \phi=0,
$$

and

$$
\begin{aligned}
\partial_{\mu}\left(\sqrt{-g}\left(1-4 \alpha \phi^{2}\right) F^{\mu \nu}\right) & =0, \\
G_{\mu \nu} & =-8 \pi G\left(T_{\mu \nu}^{(A)}+T_{\mu \nu}^{(\phi)}\right),
\end{aligned}
$$

where the energy-momentum tensor components of the gauge field and scalar field read, respectively,

$$
\begin{gathered}
T_{\mu \nu}^{(A)}=\left(\frac{1}{4}-\alpha \phi^{2}\right)\left(F_{\mu \sigma} F_{\nu}^{\sigma}-\frac{1}{4} g_{\mu \nu} F_{\alpha \beta} F^{\alpha \beta}\right), \\
T_{\mu \nu}^{(\phi)}=\partial_{\mu} \phi \partial_{\nu} \phi-g_{\mu \nu}\left(\frac{1}{2} \partial_{\sigma} \phi \partial^{\sigma} \phi+\frac{m^{2}}{2} \phi^{2}\right) \\
-\gamma\left(g_{\mu \sigma} g_{\nu \lambda}+g_{\nu \sigma} g_{\mu \lambda}\right) \eta^{\sigma \alpha \gamma \delta} \eta^{l \lambda \kappa \rho} \mathcal{R}_{\gamma \delta \kappa \rho} D_{\alpha} D_{l}\left(\phi^{2}\right) .
\end{gathered}
$$

In this paper, we would like to discuss the scalarization of self-gravitating solutions of the Einstein-Maxwell equations. We assume staticity and cylindrical symmetry and hence choose the following Ansatz for the metric, gauge and scalar field:

$$
\begin{aligned}
\mathrm{d} s^{2} & =N^{2} \mathrm{~d} t^{2}-H^{2} \mathrm{~d} \rho^{2}-L^{2} \mathrm{~d} \varphi^{2}-K^{2} \mathrm{~d} z^{2}, \\
A_{\mu} \mathrm{d} x^{\mu} & =A(\rho) \mathrm{d} \varphi, \quad \phi=\phi(\rho)
\end{aligned}
$$

where the metric functions $N, H, J, K$ depend only on $\rho$. In the following, we will now fix the gauge by imposing $H(\rho)=1$, which implies $K(\rho) \equiv N(\rho)$. Inserting the Ansatz into the equations of motion (2.3), (2.4) we note that the Maxwell equation can be integrated separately, leading to

$$
A^{\prime}=B_{0} \frac{L}{\left(1-4 \alpha \phi^{2}\right) N^{2}},
$$

where $B_{0}$ is an integration constant. The magnetic field of the solution, which points in the direction of the $z$ axis, is then given by

$$
\mathcal{B}=-\frac{A^{\prime}}{L}=-\frac{B_{0}}{\left(1-4 \alpha \phi^{2}\right) N^{2}} .
$$

The remaining equations read

$$
\begin{gathered}
\frac{N^{\prime \prime}}{N}+\frac{\left(N^{\prime}\right)^{2}}{2 N^{2}}=\frac{\kappa}{4}\left(-\epsilon_{s}+\epsilon_{v}-U\right)+4 \kappa \gamma \mathcal{F}_{1}, \\
\frac{L^{\prime \prime}}{L}+\frac{L^{\prime} N^{\prime}}{L N}-\frac{\left(N^{\prime}\right)^{2}}{2 N^{2}}=\frac{\kappa}{4}\left(-\epsilon_{s}-3 \epsilon_{v}-U\right)-4 \kappa \gamma \mathcal{F}_{2},
\end{gathered}
$$




$$
\begin{aligned}
& \phi^{\prime \prime}+\left(\frac{L^{\prime}}{L}+\frac{2 N^{\prime}}{N}\right) \phi^{\prime} \\
& =\phi\left(m^{2}-4 \alpha \frac{\left(A^{\prime}\right)^{2}}{L^{2}}-16 \gamma \frac{L^{\prime \prime} N^{\prime 2}+2 L^{\prime} N^{\prime} N^{\prime \prime}}{L N^{2}}\right),
\end{aligned}
$$

where we have used the following abbreviations:

$$
\begin{aligned}
\kappa & =16 \pi G, \quad \epsilon_{s}=\frac{\left(\phi^{\prime}\right)^{2}}{2}, \\
\epsilon_{v} & =\frac{\left(A^{\prime}\right)^{2}}{2 L^{2}}\left(1-4 \alpha \phi^{2}\right), \quad U=\frac{m^{2}}{2} \phi^{2},
\end{aligned}
$$

as well as

$$
\begin{aligned}
\mathcal{F}_{1}= & \frac{N^{\prime 2}}{N^{2}}\left(\phi \phi^{\prime \prime}+\phi^{\prime 2}\right)+\frac{2 N^{\prime} N^{\prime \prime}}{N^{2}} \phi \phi^{\prime}, \\
\mathcal{F}_{2}= & \left(\phi \phi^{\prime \prime}+\phi^{\prime 2}\right)\left(\frac{N^{\prime 2}}{N^{2}}-2 \frac{L^{\prime} N^{\prime}}{L N}\right) \\
& +\phi \phi^{\prime}\left(\frac{2 N^{\prime} N^{\prime \prime}}{N^{2}}-2 \frac{L^{\prime} N^{\prime \prime}}{L N}-2 \frac{L^{\prime \prime} N^{\prime}}{L N}\right)
\end{aligned}
$$

and the prime denotes derivative with respect to $\rho$. Moreover, we have a constraint, which reads

$$
\begin{aligned}
\frac{N^{\prime 2}}{2 N^{2}}+\frac{N^{\prime} L^{\prime}}{N L} & =\frac{\kappa}{4}\left(\epsilon_{s}+\epsilon_{v}-U\right)+\kappa \gamma \mathcal{F}_{3}, \\
\mathcal{F}_{3} & =12 \gamma \kappa \phi \phi^{\prime}\left(\frac{L^{\prime} N^{\prime 2}}{L N^{2}}\right) .
\end{aligned}
$$

The system has to be solved for $\rho \in[0, \infty[$ with the following boundary conditions which guarantee the regularity at origin and the localization of the solution:

$$
\begin{aligned}
& N(0)=1, \quad N^{\prime}(0)=0, \quad L(0)=0, \\
& L^{\prime}(0)=1, \quad \phi^{\prime}(0)=0, \quad \phi(\rho \rightarrow \infty)=0 .
\end{aligned}
$$

Note that $\kappa$ and $m$ can be set to unity by appropriate rescalings of the fields and of the radial variable, respectively. The equations have to be solved numerically, see Appendix B for a short summary of the numerical procedure, and we have found it convenient to add an additional boundary condition on the scalar field, namely setting $\phi(0)$ equal to a nonvanishing value. As the equations and boundary conditions make clear, this overdetermines the problem and in turn fixes one of the coupling constants in the model uniquely. We will comment more on this in the following discussion.

In the vacuum case, i.e., for $\phi \equiv 0, B_{0}=0$, the equations of motion have well-known solutions first given in [26]. For the boost-symmetric case, these are as follows:

(i) $N \sim 1, L \sim \beta \rho$ : this is a locally flat space-time which globally possesses a deficit angle $\Delta=2 \pi(1-\beta)$. The metric describes e.g., the (asymptotic) space-time of a cosmic string (see e.g., [20] and references therein).

(ii) $N \sim \rho^{2 / 3}, L \sim \rho^{-1 / 3}$ : this space-time obviously does not fulfill the regularity conditions [see (2.16)] on the axis, however, it is important in the following in the description of the space-time away from the sources of the gravitational field.

\section{A. Small and vanishing scalar field}

In the case of vanishing scalar field, i.e., for the case $\phi \equiv 0$, a combination of the equations (2.10), (2.11) and (2.15) shows that the metric functions have to fulfill $N^{\prime} \propto L$. This clearly excludes the string-type solution far away from the magnetic field, while the vacuum solution with $L \sim \rho^{-1 / 3}, N \sim \rho^{2 / 3}$ fulfills this requirement. In fact, the solution can be given in closed form and is often referred to as the magnetic Melvin universe [16]. In order to discuss the scalar field in this background, it is convenient to adopt Weyl-type coordinates with $\mathrm{d} \rho=N \mathrm{~d} r$. The solution then reads

$$
\begin{aligned}
\mathrm{d} s^{2} & =N^{2}\left(\mathrm{~d} t^{2}-\mathrm{d} r^{2}-d \varphi^{2}\right)-\frac{r^{2}}{N^{2}} \mathrm{~d} \varphi^{2}, \quad F_{r \varphi}=\frac{B_{0} r}{N^{2}}, \\
N & =\left(1+\frac{1}{4} B_{0}^{2} r^{2}\right) .
\end{aligned}
$$

The relation between $\rho$ and $r$ is

$$
\begin{aligned}
\rho & =r+\frac{1}{12} B_{0}^{2} r^{3} \\
\text { resp., } r & =\left(\frac{6 \rho}{B_{0}^{2}}(1+\sqrt{\Sigma})\right)^{1 / 3}+\left(\frac{6 \rho}{B_{0}^{2}}(1-\sqrt{\Sigma})\right)^{1 / 3} \\
\Sigma & =1+\frac{1}{27 B_{0}^{2} \rho^{2}} .
\end{aligned}
$$

In the following, we will use the coordinate $r$ to study the scalar field equation in the background of this solution. The scalar field equation [see (2.3)] then reads

$$
\begin{aligned}
& \frac{1}{r} \partial_{r}\left(r \partial_{r} \phi\right)+\left(2 \alpha F_{\mu \nu} F^{\mu \nu}+2 \gamma \mathcal{G}-m^{2}\right) N^{2} \phi=0, \\
& \mathcal{G}=\frac{\left(3 B_{0}^{4} r^{4}-24 B_{0}^{2} r^{2}+16\right) B_{0}^{4}}{4 N^{8}}, \quad F_{\mu \nu} F^{\mu \nu}=\frac{2 B_{0}^{2}}{N^{4}} .
\end{aligned}
$$

The general solution to this equation can only be found numerically, but we can understand the behavior of the solutions when looking at the asymptotic behavior of the scalar field. We will discuss the two cases $\gamma=0$ and $\alpha=0$ separately now. 


\section{1. $\gamma=0$}

For $r \ll 1$, we can approximate $N^{ \pm 2} \approx 1 \pm B_{0}^{2} r^{2} / 2$ and Eq. (2.19) becomes

$$
\begin{aligned}
& \quad \frac{1}{r} \partial_{r}\left(r \partial_{r} \phi\right)-\mathcal{A}_{0} \phi-\mathcal{A}_{2} r^{2} \phi=0, \quad \mathcal{A}_{0}=m^{2}-4 \alpha B_{0}^{2}, \\
& \mathcal{A}_{2}=\frac{m^{2} B_{0}^{2}}{2}+2 \alpha B_{0}^{4} .
\end{aligned}
$$

Introducing $z=\sqrt{\mathcal{A}_{2}} r^{2}$ and defining $\phi=\exp (-z / 2) \chi$, we obtain

$$
z \ddot{\chi}+(1-z) \dot{\chi}+\mathcal{A} \chi=0, \quad \mathcal{A}=-\left(\frac{\mathcal{A}_{0}}{4 \sqrt{\mathcal{A}_{2}}}+\frac{1}{2}\right),
$$

where the dot denotes the derivative with respect to $z$. This is the confluent hypergeometric equation that has as suitable solutions the Laguerre polynomials $\chi(z) \sim \mathcal{L}_{\mathcal{A}}$. Hence for small $r$ Eq. (2.20) has the solution

$$
\phi(r \ll 1)=\phi_{0} \exp \left(-\frac{\sqrt{\mathcal{A}_{2}}}{2} r^{2}\right) \mathcal{L}_{\mathcal{A}}\left(\sqrt{\mathcal{A}_{2}} r^{2}\right)
$$

For $\mathcal{A} \in \mathbb{N}$ the $\mathcal{L}_{\mathcal{A}}$ possess a number of nodes. This suggests that we should also be able to construct scalar field solutions that possess a number $k$ of nodes, a conclusion that we have confirmed by an explicit numerical construction, see below. In fact, using these arguments, we can give a rough approximation of the critical value of $\alpha$ to obtain solutions. From the requirement that $\mathcal{A}=k$, $k=0,1,2, \ldots$, we find that

$$
\alpha \gtrsim \frac{m^{2}}{4 B_{0}^{2}} \quad \text { for } k=0,1,2,3, \ldots
$$

For $r \gg 1$ we introduce $y=r^{3}$ and Eq. (2.19) becomes a modified Bessel equation of the form

$$
y^{2} \frac{\mathrm{d}^{2} \phi}{\mathrm{d} y^{2}}+y \frac{\mathrm{d} \phi}{\mathrm{d} y}-\frac{m^{2} B_{0}^{4}}{144} y^{2} \phi=0
$$

such that the asymptotic decay of the solution is

$$
\phi(r \gg 1) \sim K_{0}\left(\frac{m B_{0}^{2}}{12} r^{3}\right) \sim r^{-3 / 2} \exp \left(-r^{3}\right) .
$$

This analysis also clearly demonstrates why it is necessary to have a mass term for the scalar field. For $m=0$, as is well known, the scalar field would behave like $\phi(r) \sim \ln (r)$ asymptotically and would hence not be localized.

\section{2. $\alpha=0$}

In this case Eq. (2.19) becomes

$$
\begin{gathered}
\frac{1}{r} \partial_{r}\left(r \partial_{r} \phi\right)-\mathcal{C}_{0} \phi-\mathcal{C}_{2} r^{2} \phi=0, \\
\mathcal{C}_{0}=m^{2}-8 \gamma B_{0}^{4}, \quad \mathcal{C}_{2}=\frac{m^{2} B_{0}^{2}}{2}+24 \gamma B_{0}^{6} .
\end{gathered}
$$

With similar substitutions as above, we find

$$
\begin{aligned}
\phi(r \ll 1) & =\phi_{0} \exp \left(-\frac{\sqrt{\mathcal{C}_{2}}}{2} r^{2}\right) \mathcal{L}_{\mathcal{C}}\left(\sqrt{\mathcal{C}_{2}} r^{2}\right), \\
\mathcal{C} & =-\left(\frac{\mathcal{C}_{0}}{4 \sqrt{\mathcal{C}_{2}}}+\frac{1}{2}\right)
\end{aligned}
$$

Again, the analysis suggests that radially excited solutions should be present and we can give a rough approximation of the critical value of $\gamma$ to obtain solutions. From the requirement that $\mathcal{C}=k, k=0,1,2, \ldots$, we find that

$$
\gamma \gtrsim \frac{m^{2}}{8 B_{0}^{4}} \quad \text { for } k=0,1,2,3, \ldots
$$

For $r \gg 1$ the behavior is exactly as in the $\gamma=0$ because it is the mass term that determines the asymptotic regime in both cases.

\section{SCALAR-MAGNETIC FIELD COUPLING}

Here, we would like to discuss the case $\gamma=0$, i.e., we consider only the nonminimal coupling between the gauge field and the scalar field. As stated above, we can choose appropriate scalings to set $\kappa=m \equiv 1$ without losing generality. The parameters to be varied in the following are then the nonminimal coupling constant $\alpha$ and the absolute value of the magnetic field strength $B_{0}$. As discussed above for small scalar fields, we expect solutions with scalar field nodes to be present in our system. We, indeed, have confirmed this numerically. In Fig. 1, we compare the analytical expression (2.22) (denoted $\bar{\phi}$ and given in solid) with the numerical solutions of the full set of equations for $B_{0}=1$ and $\phi(0)=0.01$ (dashed) for the solution with no nodes $(k=0)$ and that with one node $(k=1)$. As expected, the approximation is not perfect, but gives a good idea of the qualitative behavior of the functions. We also find that the approximation gives a good order of magnitude approximation of the location of the zeros of the scalar field function. We give some values for the location of the nodes of the $k=2$ solution, i.e., the solution with two nodes, in comparison to the location of the zeros of the second Laguerre polynomial $\mathcal{L}_{2}$ in Table I.

As such the $k=0$ and $k=1$ in Fig. 2, respectively, refers to the solution that has no nodes in the scalar field $(k=0)$ and that which has one node $(k=1)$. Moreover, (2.12) suggests that a tachyonic instability appears in the 


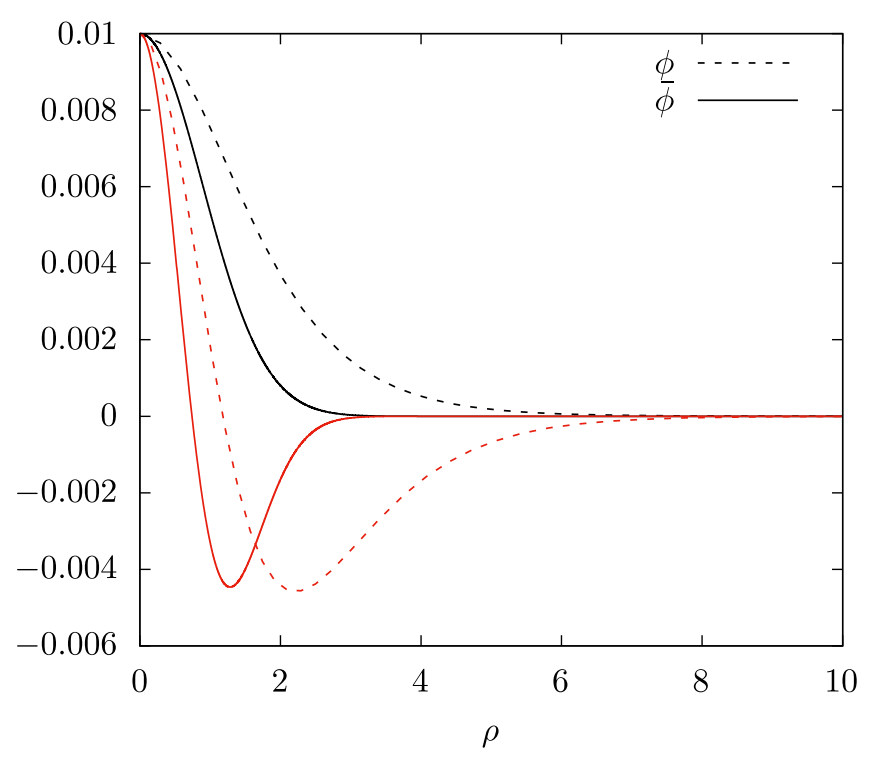

FIG. 1. We compare the profiles of the analytical approximation $\bar{\phi}$ [see (2.22)] (solid) for the scalar field with the full numerical solution $\phi$ (dashed) for $k=0$ (black) and $k=1$ (red) nodes for $B_{0}=1.0$ and $\phi(0)=0.01$. These choices correspond to $\alpha=0.55$ for $k=0$ and $\alpha=1.40$ in the case $k=1$, respectively. Note that we have used $\rho \approx r$ for this plot, which is a good approximation for small values of the radial coordinate.

system only for $\alpha>0$. From (2.23), we know that $\alpha \gtrsim \frac{m^{2}}{4 B_{0}}$. The curve $\alpha=\frac{m^{2}}{4 B_{0}}=\frac{1}{4 B_{0}}$ (remember $m \equiv 1$ ) is given in Fig. 2 (blue, dotted-dashed) together with the numerically determined values of $\alpha_{0}$ (solid) and $\alpha_{\mathrm{cr}}$ (dashed) which determine the interval in $\alpha$ for which nontrivial scalar field solutions exist for a given value of $B_{0}$. Here, the value of $\alpha_{\mathrm{cr}}$ is given by the observation that there exists a value of $\alpha=1 /\left(4 \phi(0)^{2}\right)$ for which the magnetic field on the axis of symmetry [see (2.9)] diverges. Solutions exist for $\alpha>\alpha_{\mathrm{cr}}$. That this is closely related to the fact that $\alpha$ needs to be sufficiently large to generate a tachyonic instability can be seen by noting that the curve $\alpha=1 /\left(4 \phi(0)^{2}\right)$ is a good approximation to the $\alpha_{\text {cr }}$ curve for $k=0$ and small $\alpha$.

Increasing $\alpha$ too strongly, the scalar field can no longer be nontrivial in the space-time and becomes identically zero due to the backreaction of the space-time. This value

TABLE I. We give the location of the zeros of the scalar field solution with $k=2$ notes, $\rho_{0}^{(1)}$ and $\rho_{0}^{(2)}$, for some values of $B_{0}$ and $\alpha$ and compare them with the zeros of the corresponding Laguerre polynomial $\mathcal{L}_{2}, \bar{\rho}_{0}^{(1)}$ and $\bar{\rho}_{0}^{(2)}$, respectively.

\begin{tabular}{lrllll}
\hline \hline$B_{0}$ & \multicolumn{1}{c}{$\alpha$} & $\rho_{0}^{(1)}$ & $\rho_{0}^{(2)}$ & $\bar{\rho}_{0}^{(1)}$ & \multicolumn{1}{c}{$\bar{\rho}_{0}^{(2)}$} \\
\hline 0.05 & 124.2 & 4.1 & 11.5 & 2.6 & 15.5 \\
0.07 & 69.5 & 3.8 & 9.6 & 2.1 & 13.2 \\
0.2 & 13.7 & 2.3 & 5.5 & 1.2 & 7.5 \\
0.4 & 5.8 & 1.5 & 3.8 & 0.8 & 5.5 \\
0.5 & 4.6 & 1.3 & 3.3 & 0.6 & 4.8 \\
\hline \hline
\end{tabular}

of $\alpha$ is denoted by $\alpha_{0}$. Note that had we only studied the scalar field in the background of the Melvin universe, the two limiting values would be equal $\alpha_{0}=\alpha_{\mathrm{cr}}$. However, here, the backreaction of the scalar field on the space-time leads to the observation that nontrivial scalar field solutions exist in a given interval of $\alpha$ (for a given $B_{0}$ ) rather than for a sole value of $\alpha$. We observe that the interval in $\alpha$ increases with increasing magnetic field strength $B_{0}$. For $B_{0} \rightarrow 0$, our numerical results indicate that the interval shrinks to zero and both $\alpha_{\mathrm{cr}}$ as well as $\alpha_{0}$ tend to infinity. This makes sense since the vanishing $B_{0}$ limit corresponds to Minkowski space-time (in cylindrical coordinates) and this space-time cannot be scalarized.

Comparing the scalar field solutions with a different number of nodes $k$, we observe that for a given $B_{0}$, we have to choose $\alpha$ larger to find $k=1$ solutions than $k=0$ solutions. Interestingly, the ranges of $\alpha$ for which $k=0$ and $k=1$ solutions exist, respectively, do not overlap in the range of values of $B_{0}$ that we have studied here. To state it differently, fixing $B_{0}$ and $\alpha$ within the range of values given in Fig. 2 we will either obtain a scalar field solution with no nodes or one with one node, but not both at the same time. Hence, the solutions with nodes cannot really be interpreted as the radially excited solution of the fundamental ones, as is often done in other nonlinear, self-gravitating systems.

Naturally, the question arises whether we are simply not able to find the fundamental $k=0$ solutions for the range of parameters for which the $k=1$ solutions exist. Due to the numerical procedure that we have employed, we are certain that this is not the case. We have fixed the value of the scalar field at the origin, $\phi(0)$, which is not an independent boundary condition. The choice of this value hence fixes the value of one of the couplings in the model uniquely and we have chosen the value of $\alpha$ to be computed by our numerical procedure. As such, we have followed the evolution of the branch of solutions with no nodes and that with one node and determined the values of $\alpha$ at given $B_{0}$ for which these solutions exist.

\section{SCALAR-CURVATURE COUPLING}

Here, we will set $\alpha=0$, i.e., we consider only the nonminimal coupling between the Gauss-Bonnet term and the scalar field. In this case, the equations of motion (2.3)-(2.12) have to be diagonalized with respect to the second derivatives, see Appendix A for details.

We observe that nontrivial scalar field solutions exist only for sufficiently large values of the nonminimal coupling $\gamma$, i.e., for $\gamma>\gamma_{0}$. The dependence of this value on $B_{0}$ is shown in Fig. 2 (solid lines) for scalar field solutions with no nodes $(k=0$, black) and one node ( $k=0$, black), respectively. Again, we observe that one has to choose the nonminimal coupling large in order to obtain solutions with $k=1$ as compared to the $k=0$ case. Interestingly, in this case, the analytical expression for $\gamma_{0}$ given by $1 /\left(8 B_{0}^{4}\right)$ is not as good as in the scalar-magnetic 

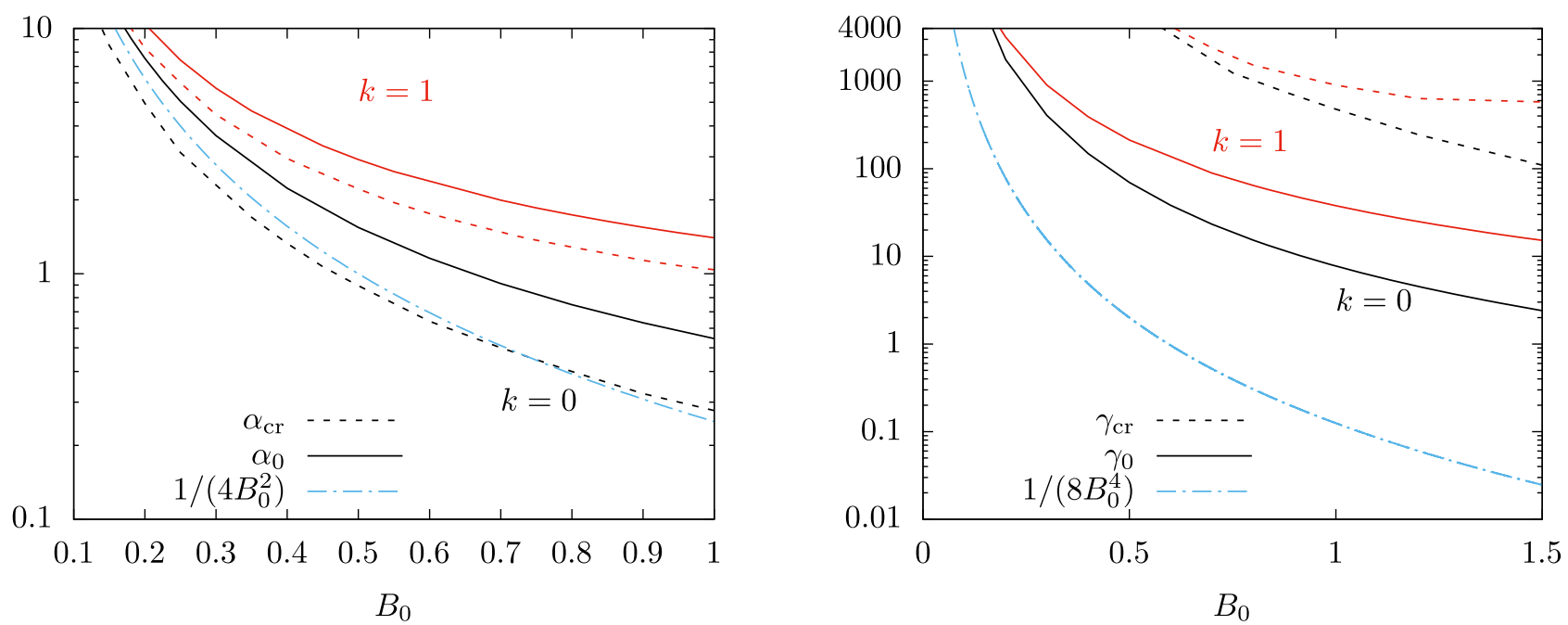

FIG. 2. Left: values of $\alpha_{0}$ (solid) and $\alpha_{\text {cr }}$ (dashed) between which the scalarized solutions with $k=0$ (black) and $k=1$ (red) nodes, respectively, exist in dependence on the magnetic field parameter $B_{0}$ for $\gamma=0$. We also give $1 /\left(4 B_{0}^{2}\right)$ (dotted-dashed, blue) which is a rough approximation of the critical value of $\alpha$ (see text for details). Right: values of $\gamma_{0}$ (solid) and $\gamma_{\mathrm{cr}}$ (dashed) between which the scalarized solutions with $k=0$ (black) and $k=1$ (red) nodes, respectively, exist in dependence on the magnetic field parameter $B_{0}$ for $\alpha=0$. We also give $1 /\left(8 B_{0}^{4}\right)$ (dotted-dashed, blue) which is a rough approximation of the critical value of $\alpha$ (see text for details).

field coupling case. This is likely related to the fact that the space-time background approximation is not a good approximation in this case as the scalar field is nonminimally coupled to the curvature. When increasing the coupling $\gamma$, the scalar field increases in absolute value, which leads to increased backreaction of the scalar field on the space-time. In fact, we observe that there exists a maximal value of $\phi(0)$, or equivalently a maximal value of $\gamma=\gamma_{\mathrm{cr}}$ beyond which no scalarized solutions exist anymore. This is true for both the $k=0$ and the $k=1$ case. The values of $\gamma_{\mathrm{cr}}$ in dependence on $B_{0}$ are shown in Fig. 2 (dashed lines). Note that for the scalar-curvature case the critical value of the coupling is always larger than the value where the scalar field vanishes identically, while for the

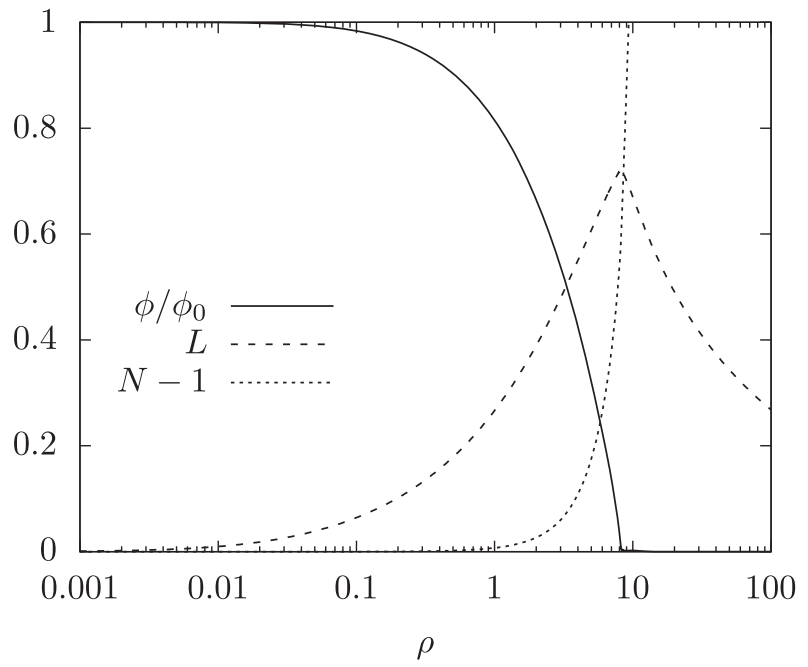

scalar-magnetic field coupling, this is exactly opposite. This is related to the fact that the scalar field directly sources these fields and hence leads to an increased repulsive effect for the magnetic fields and an increased attractive effect in the case of the curvature fields. This also demonstrates that the two couplings are qualitatively different in nature. Another difference to the scalarmagnetic field coupling is that the domain of existence of scalarized solutions for $\gamma \in\left[\gamma_{0}: \gamma_{\mathrm{cr}}\right]$ is now not mutually exclusive for different node solutions. The range of $\gamma$ for $k=0$ overlaps partially with the range of $\gamma$ for $k=1$, as Fig. 2 clearly demonstrates, and in this overlapping region the $k=1$ solutions can be interpreted as the radially excited version of the $k=0$ solution.

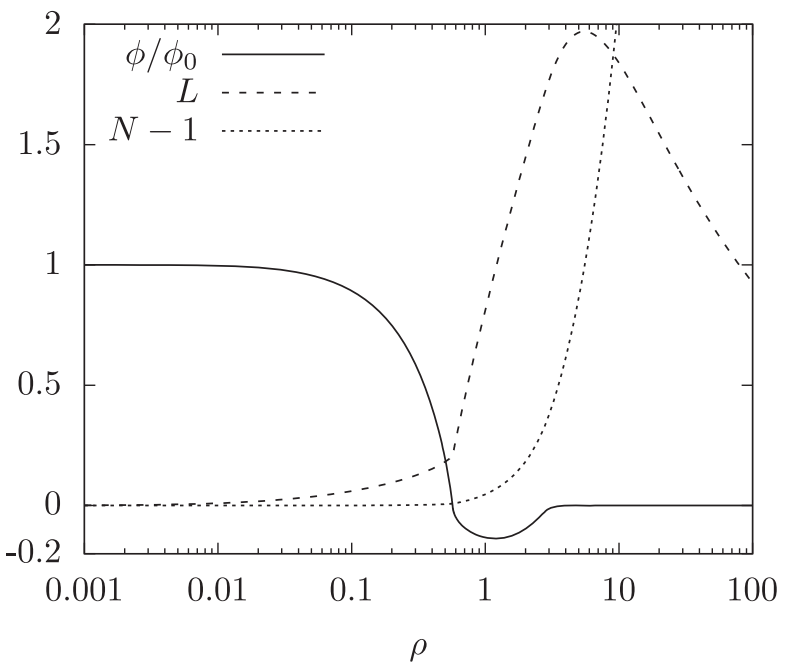

FIG. 3. Profiles of the scalar field function $\phi / \phi_{0}$ and the metric functions $N$ and $L$ for a value of $\gamma$ close $\gamma_{\text {cr }}$ for $B_{0}=1$ and $k=0$ (left) and $k=1$ (right), respectively. 


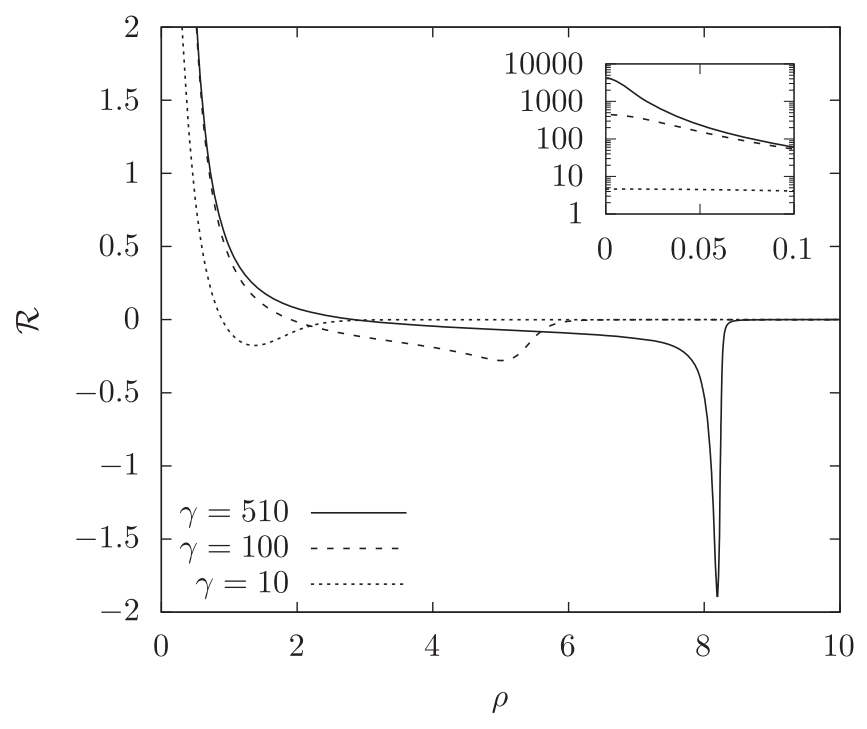

FIG. 4. Ricci scalar $\mathcal{R}$ of the scalarized Melvin solution for $k=0, B_{0}=1$ and for different values of $\gamma$ including $\gamma=510$ close to $\gamma_{\mathrm{cr}}$.

Finally, we would like to discuss why the solutions cease to exist at $\gamma=\gamma_{\mathrm{cr}}$. A solution close to the limiting solution is shown in Fig. 3 for $k=0$ and $k=1$, respectively. Clearly, the scalar field becomes zero outside of a sharply defined radius $\rho_{\text {cr }}$ such that at $\rho=\rho_{\text {cr }}$ the scalar field function is nondifferentiable. For $\rho>\rho_{\text {cr }}$ the solution corresponds to the Melvin magnetic universe, while it possesses a nontrivial, scalarized interior.

We observe that the Ricci scalar $\mathcal{R}$ increases strongly on the symmetry axis of the solution when approaching $\gamma_{\mathrm{cr}}$. This is demonstrated for the $k=0$ solution with $B_{0}=1$ in Fig. 4, where we give $\mathcal{R}$ for $\gamma=10$ (close to $\gamma_{0}$ ), an intermediate $\gamma=100$ as well as for $\gamma=510$ (close to $\gamma_{\mathrm{cr}}$ ). The subfigure of Fig. 4 shows the strong increase of $\mathcal{R}$ at $\rho=0$. The figure further demonstrates that the Ricci scalar becomes discontinuous at $\rho=\rho_{\text {cr }}$ indicating that the limiting space-times possess singularities.

\section{CONCLUSIONS}

In this paper, we have demonstrated that the process of spontaneous scalarization is not specific to compact objects such as black holes, neutron stars or boson stars, but also exists for extended self-gravitating solutions. We have used the Melvin magnetic universe solution that describes magnetic fields orientated into the direction of the symmetry axis and possesses a cylindrically symmetric, static space-time. In the small scalar field limit, we find that the linear Klein-Gordon equation of a massive, real scalar field leads to solutions described by the Laguerre polynomials close to the axis of symmetry and by Bessel functions asymptotically, respectively. These results suggest that a discrete family of scalar field solutions exists in the model. We have demonstrated this by explicit numerical construction of the solutions including backreaction of the space-time and the magnetic field, respectively. The solutions are characterized by two parameters: the magnetic field parameter $B_{0}$ and the value of the scalar field on the symmetry axis, which is linked to the value of the nonminimal coupling. The scalarized solutions, which are nontrivial deformations of the underlying Melvin magnetic universe solution, exist only in specific intervals of the nonminimal couplings. Since these intervals for different node numbers are in some cases mutually exclusive, the question arises whether the higher node solutions can be interpreted as radial excitations of the fundamental solutions with no nodes, as is often done in self-gravitating systems.

It would be interesting to understand whether such scalarization processes of extended magnetic fields could be observed in the universe and if not, if observations could provide limits on the coupling parameters or even exclude extended gravity models with nonminimal coupling terms. An interesting future investigation could be another very specific cylindrically symmetric system that possesses magnetic fields: the cosmic string. While the outside of a cosmic string is characterized by a massive gauge field and a massive scalar field (spontaneously broken phase), the inside of the string core remains in the symmetric, i.e., false vacuum of the model in which the gauge symmetry is unbroken. It is surely of interest to understand whether cosmic strings that are hypothetical relics of the primordial universe could be scalarized spontaneously and, if so, how this would change the properties of these objects.

In order to get an estimate of the fields and the strength of the couplings that we have used here, note that in dimensionful notation the Lagrangian density (without scalar field) should read $\mathcal{L}=\frac{c^{4}}{16 \pi G} \mathcal{R}-\frac{1}{4 \mu_{0}} F_{\mu \nu} F^{\mu \nu}$, where $\mu_{0}$ denotes the vacuum permeability and $c$ is the speed of light in vacuum. Using meter-kilogram-second units, this tells us that the "natural unit" in which the magnetic field strength is measured is roughly $10^{-18}$ Tesla, i.e., $10^{-14}$ Gauss. In our paper, we have extensively studied values of $B_{0}$ that are on the order of unity, but we have also checked that the phenomenon of spontaneous scalarization persists when increasing the order of magnitude of $B_{0}$. Since we believe that the Melvin solution can only act as a toy model for radio relics, our study has been qualitative rather than quantitative, but we are quite certain that spontaneous scalarization of extended magnetic fields with magnetic field strength much higher and on the order of that appearing in radio relics (a few micro-Gauss) is feasible.

\section{ACKNOWLEDGMENTS}

R. C. thanks CAPES for financial support under Grant No. 88887.371717/2019-00. B. H. would like to thank FAPESP for financial support under Grant No. 2019/ 01511-5 as well as the DFG Research Training Group 1620 Models of Gravity for financial support. 


\section{APPENDIX A: DIAGONALIZED EQUATIONS \\ OF MOTION}

The diagonalized equations of motion (2.3)-(2.21) read

$$
N^{\prime \prime}=\frac{F_{1}}{\mathcal{H}}, \quad L^{\prime \prime}=\frac{F_{2}}{\mathcal{H}}, \quad \phi^{\prime \prime}=\frac{F_{3}}{\mathcal{H}}
$$

with

$$
\begin{aligned}
\mathcal{H}= & 8 \kappa N^{3}\left[64\left(\left(L N^{\prime}-4 L^{\prime} N\right) N^{\prime}-\kappa L N^{2} \phi^{\prime 2}\right) \gamma^{2} \kappa N^{\prime 2} \phi^{2}+1536 \gamma^{3} \kappa^{2} L^{\prime} N^{\prime 4} \phi^{3} \phi^{\prime}+16 \gamma \kappa L N^{3} N^{\prime} \phi \phi^{\prime}-L N^{4}\right] \\
F_{1}= & {\left[128\left[\left(\left(2 N \phi^{\prime}-N^{\prime} \phi\right) m^{2} \phi^{2}+\left(2 N \phi^{\prime}-5 N^{\prime} \phi\right) \phi^{\prime 2}\right) L+\left(m^{2} \phi^{2}-\phi^{\prime 2}\right) L^{\prime} N \phi\right] \gamma^{2} \kappa^{2} N^{\prime 3} \phi\right.} \\
& +\left(\left(m^{2} \phi^{2}+\phi^{\prime 2}\right) \kappa N^{2}+4 N^{\prime 2}\right) L N^{2}-8\left(4\left[\left(\left(N \phi^{\prime}-N^{\prime} \phi\right) \phi^{\prime}+m^{2} N \phi^{2}\right) L-L^{\prime} N \phi \phi^{\prime}\right] N^{\prime}\right. \\
& \left.\left.+\left(m^{2} \phi^{2}+\phi^{\prime 2}\right) \kappa L N^{2} \phi \phi^{\prime}\right) \gamma \kappa N N^{\prime}\right] \kappa N^{4}+\left[\left(8 \gamma \kappa N^{\prime} \phi \phi^{\prime}-N\right) L N^{3}-128\left(L N^{\prime}+L^{\prime} N\right) \gamma^{2} \kappa N^{\prime 3} \phi^{2}\right] B_{0}^{2}, \\
F_{2}= & \left(8 \left(4\left(\left(\left(N \phi^{\prime}-2 N^{\prime} \phi\right) \phi^{\prime}+m^{2} N \phi^{2}\right) L^{2} N^{\prime}+2 L^{\prime 2} N^{2} \phi \phi^{\prime}-2\left(\left(N \phi^{\prime}-N^{\prime} \phi\right) \phi^{\prime}+m^{2} N \phi^{2}\right) L^{\prime} N\right) N^{\prime}\right.\right. \\
& \left.-\left(2 L N^{\prime}-L^{\prime} N\right)\left(m^{2} \phi^{2}+\phi^{\prime 2}\right) \kappa L N^{2} \phi \phi^{\prime}\right) \gamma \kappa+256\left(\left(\left(N \phi^{\prime}+N^{\prime} \phi\right) m^{2} \phi^{2}+\left(N \phi^{\prime}-N^{\prime} \phi\right) \phi^{\prime 2}\right) L\right. \\
& \left.\left.-\left(m^{2} \phi^{2}+2 \phi^{\prime 2}\right) L^{\prime} N \phi\right) \gamma^{2} \kappa^{2} L^{\prime} N^{\prime 2} \phi-\left(4\left(L N^{\prime}-2 L^{\prime} N\right) N^{\prime}-\left(m^{2} \phi^{2}+\phi^{\prime 2}\right) \kappa L N^{2}\right) L N\right) \kappa N^{4} \\
& +\left(256\left(L N^{\prime}+L^{\prime} N\right) \gamma^{2} \kappa L^{\prime} N^{\prime 2} \phi^{2}+3 L^{2} N^{3}-8\left(2 L N^{\prime}+L^{\prime} N\right) \gamma \kappa L N^{2} \phi \phi^{\prime}\right) B_{0}^{2}, \\
F_{3}=-8\left[\left(1 6 \left(\left(2\left(\left(2 N \phi^{\prime}-N^{\prime} \phi\right) m^{2} \phi-5 N^{\prime} \phi^{\prime 2}\right) L-\left(m^{2} \phi^{2}+5 \phi^{\prime 2}\right) L^{\prime} N\right) \kappa N \phi\right.\right.\right. & \\
- & \left.4\left(\left(4 N \phi^{\prime}+3 N^{\prime} \phi\right) L^{\prime}-L N^{\prime} \phi^{\prime}\right) N^{\prime}\right) \gamma^{2} \kappa N^{\prime 2} \phi \phi^{\prime}+\left(\left(m^{2} N \phi-2 N^{\prime} \phi^{\prime}\right) L-L^{\prime} N \phi^{\prime}\right) N^{3}+1536 \gamma^{3} \kappa^{2} L^{\prime} N^{\prime 4} \phi^{2} \phi^{\prime 3} \\
- & \left.2\left(\left(\left(\left(8 N \phi^{\prime}-N^{\prime} \phi\right) m^{2} \phi-17 N^{\prime} \phi^{\prime 2}\right) L-2\left(m^{2} \phi^{2}+5 \phi^{\prime 2}\right) L^{\prime} N\right) \kappa N^{2}+4\left(L N^{\prime}-4 L^{\prime} N\right) N^{\prime 2}\right) \gamma N^{\prime} \phi\right) \kappa N^{3} \\
+ & \left.2\left(\left(3 L N^{\prime}-2 L^{\prime} N\right) N-8\left(2 L N^{\prime}-L^{\prime} N\right) \gamma \kappa N^{\prime} \phi \phi^{\prime}\right) B_{0}^{2} \gamma N^{\prime} \phi\right] .
\end{aligned}
$$

\section{APPENDIX B: NUMERICAL PROCEDURE}

We have used the collocation solver COLSYS [27] for our numerical calculations. The key numerical procedure used is that of the Newton method that relies on the linearization of the problem at given points on the interval $r \in\left[0: r_{\max }\right]$ with $r_{\max }$ sufficiently large to capture the asymptotic behavior of the solutions correctly. Boundary conditions that relate to the requirements of the physical problem are employed at $r=0$ and $r=r_{\max }$ and the subsequent solution is interpolated using a spline collocation at Gaussian points. COLSYS possesses a mesh adaptation, i.e., the linearized problem is solved on a sequence of meshes with $N$ points such that $0=r_{1}<r_{2} \ldots<r_{N+1}=r_{\max }$ until a given accuracy is reached. We typically specify the error tolerance to be between $10^{-2}$ and $10^{-4}$ with the final solutions, however, having much better absolute error on the order of $10^{-6}$ to $10^{-8}$.
[1] C. A. R. Herdeiro and E. Radu, Asymptotically flat black holes with scalar hair: A review, Int. J. Mod. Phys. D 24, 1542014 (2015).

[2] T. P. Sotiriou, Black holes and scalar fields, Classical Quantum Gravity 32, 214002 (2015).

[3] D. D. Doneva and S. S. Yazadjiev, New Gauss-Bonnet Black Holes with Curvature Induced Scalarization in the Extended Scalar-Tensor Theories, Phys. Rev. Lett. 120, 131103 (2018).
[4] H. O. Silva, J. Sakstein, L. Gualtieri, T. P. Sotiriou, and E. Berti, Spontaneous Scalarization of Black Holes and Compact Stars from a Gauss-Bonnet Coupling, Phys. Rev. Lett. 120, 131104 (2018).

[5] G. Antoniou, A. Bakopoulos, and P. Kanti, Evasion of NoHair Theorems and Novel Black-Hole Solutions in GaussBonnet Theories, Phys. Rev. Lett. 120, 131102 (2018).

[6] F. M. Ramazanoğlu, Spontaneous growth of vector fields in gravity, Phys. Rev. D 96, 064009 (2017). 
[7] F. M. Ramazanoğlu, Spontaneous growth of gauge fields in gravity through the Higgs mechanism, Phys. Rev. D 98, 044013 (2018).

[8] F. M. Ramazanoğlu, Spontaneous tensorization from curvature coupling and beyond, Phys. Rev. D 99, 084015 (2019).

[9] S. Barton, B. Hartmann, B. Kleihaus, and J. Kunz, Spontaneously vectorized Einstein-Gauss-Bonnet black holes, Phys. Lett. B 817, 136336 (2021).

[10] A. W. Whinnett, Spontaneous scalarization and boson stars, Phys. Rev. D 61, 124014 (2000).

[11] M. Alcubierre, J. C. Degollado, D. Nunez, M. Ruiz, and M. Salgado, Dynamic transition to spontaneous scalarization in boson stars, Phys. Rev. D 81, 124018 (2010).

[12] Y. Brihaye and B. Hartmann, Spontaneous scalarization of boson stars, J. High Energy Phys. 09 (2019) 049.

[13] T. Damour and G. Esposito-Farese, Nonperturbative Strong Field Effects in Tensor-Scalar Theories of Gravitation, Phys. Rev. Lett. 70, 2220 (1993).

[14] P. Chen, T. Suyama, and J. Yokoyama, Spontaneous scalarization: Asymmetron as dark matter, Phys. Rev. D 92, 124016 (2015).

[15] T. Anson, E. Babichev, C. Charmousis, and S. Ramazanov, Cosmological instability of scalar-Gauss-Bonnet theories exhibiting scalarization, J. Cosmol. Astropart. Phys. 06 (2019) 023.

[16] M. A. Melvin, Pure magnetic and electric geons, Phys. Lett. 8, 65 (1964).

[17] K.S. Thorne, Absolute stability of Melvin's magnetic universe, Phys. Rev. 139, B244 (1965).

[18] N. M. Santos and C. A. R. Herdeiro, Black holes, stationary clouds and magnetic fields, Phys. Lett. B 815, 136142 (2021).
[19] K. Bronnikov, N. O. Santos, and A. Wang, Cylindrical systems in general relativity, Classical Quantum Gravity 37, 113002 (2020).

[20] M. B. Hindmarsh and T. W. B. Kibble, Cosmic strings, Rep. Prog. Phys. 58, 477 (1995).

[21] M. Kierdorf, R. Beck, M. Hoeft, U. Klein, R. J. van Weeren, W. R. Forman, and C. Jones, Relics in galaxy clusters at high radio frequencies, Astron. Astrophys. 600, A18 (2017).

[22] J. C Kempner, E. L. Blanton, T. E. Clarke, T. A. Enßlin, M. Johnston-Hollitt, and L. Rudnick, Conference note: A taxonomy of extended radio sources in clusters of galaxies, in The Riddle of Cooling Flows in Galaxies and Clusters of galaxies, edited by T. Reiprich, J. Kempner, and N. Soker (Springer, Dordrecht, The Netherlands, 2004), p. 335.

[23] M. Kierdorf, R. Beck, M. Hoeft, U. Klein, R. J. van Weeren, W. R. Forman, and C. Jones, Relics in galaxy clusters at high radio frequencies, Astron. Astrophys. 600, A18 (2017).

[24] R. van Weeren, M. Brüggen, H. J. A. Röttgering, and M. Hoeft, Using double radio relics to constrain galaxy cluster mergers: A model of double radio relics in CIZA J2242. $8+$ 5301, Mon. Not. R. Astron. Soc. 418, 230 (2011).

[25] S. E. Nuza, M. Hoeft, S. Göttloeber, R. van Weeren, and G. Yepes, Radio relics in the MareNostrum Universe, Mem. Soc. Astron. Ital. 82, 674 (2011).

[26] T. Levi-Civita, (1919): ds2 einsteiniani in campi newtoniani. IX: L'analogo del potenziale logaritmico, Rend. Accad. Lincei 28, 101 (1919).

[27] U. Ascher, J. Christiansen, and R. D. Russell, A collocation solver for mixed order systems of boundary value problems, Math. Comput. 33 (1979) 659; ACM Trans. 7, 209 (1981). 